\title{
The risk factors related to operative duration and its relationship with clinical outcome in pediatric patients undergone RouX-en-Y hepaticojejunostomy
}

Ang Li

Chongqing Medical University Affiliated Children's Hospital

Kai Gao

Chongqing Medical University Affiliated Children's Hospital

Qingshuang Liu

Chongqing Medical University Affiliated Children's Hospital

Jingyu Chen

Chongqing Medical University Affiliated Children's Hospital

Chunbao Guo ( $\nabla$ guochunbao@foxmail.com )

Chongqing Medical University https://orcid.org/0000-0002-9132-3096

Research article

Keywords: Operative time, Roux-en-Y hepaticojejunostomy, Postoperative recovery, Perioperative complications

Posted Date: March 30th, 2020

DOI: https://doi.org/10.21203/rs.3.rs-19847/v1

License: (c) (1) This work is licensed under a Creative Commons Attribution 4.0 International License.

Read Full License 


\section{Abstract}

Background: The operative duration might be important for perioperative morbidity and the involvement has not been fully characterized in pediatric patients. We here determined the perioperative variables in association with operative duration and its influence on the clinical outcome in the pediatric patients.

Methods: We retrospectively reviewed 701 patients who underwent elective choledochal cysts followed by Roux-en-Y hepaticojejunostomy between August 2010 and Aug 2019. Based on the median operating time (165 min), patients were separated into two groups, including a long-time group (>165 min) and a short-time group ( $<165 \mathrm{~min}$ ). The independent risk factors for the operative time were determined usng the multivariable logistic regression analyses. To adjust for any potential selection bios, propensity score matching was performed for the long-time and short-time groups. In the 192 matched patients, perioperative outcomes, including postoperative recovery, complications measurement, and length of hospital stay were analyzed.

Results: The operating time was often increased by excision difficulty caused by the lager choledochal cyst size (OR=1.56; 95\% Cl, 1.09-2.23; $p<0.001)$, the greater $\mathrm{BMI}(\mathrm{OR}=1.02 ; 95 \% \mathrm{Cl}, 1.00-1.15$; $\mathrm{p}=0.018)$, older age $(\mathrm{OR}=1.17 ; 95 \% \mathrm{Cl}, 1.02-1.39 ; \mathrm{p}=0.012)$ under multivariate analysis. The long-time surgical duration was associated with delay for gastrointestinal functional recovery, measured with the first defecation ( $p=0.027)$ and first bowel movement $(p=0.019)$. Moreover, a significant decrease in serum albumin were presented in the long-time group compared with the short time group $(p=0.0035)$. The total length of postoperative hospital stay was longer in patients with long-time group $(7.51 \pm 2.03$ days) than patients with short-time group $(6.72 \pm 1.54$ days, $p=0.006)$.

Conclusions: Our data demonstrated that short operating time was associated with favorable postoperative results. The influencing factors for operating time could be ameliorated in the perioperative period to gain a better outcome after major abdominal operations.

\section{Introduction}

During surgical procedures, longer operative time might lead to delayed recovery and be associated with more postoperative complications[1, 2]. In the general surgery literature, particularly in gastroenterological surgery, longer operative time might result in delayed gastroinstinal function recovery, postoperative intestinal obstruction, and prolonged hospitalization [3, 4]. It is therefore recommended to optimize the speed of the surgery, especially for the major classical operation, to maintain an appropriate postoperative recovery and improve postoperative outcomes. In order to assess the impact of operative time on postoperative outcomes, it must be analyzed the risk factors that contribute to longer operative duration and determined its contribution to surgical outcome.

Operative times have been used as a measure of competence for surgeon. The specific surgeon properties, involved in operative time are well documented in the literature, which have been used to determine operative learning curves[5,6]. A long operating time may involve insufficient experience of 
surgeons. Individual surgeon characteristics should account for a fairly large part of the variability in operative time[7, 8]. In addition, a long operating time was associated with a surgeon who is more tired and may lose focus. On the other hand, operation with rush might be associated with increase the occurrence of errors during surgery. There are many other factors that may potentially impact upon operative duration including the surgeon's operative experience, the actual pathology undertaken[9]. A clear evaluation the factors, accounting for the operative duration is currently lacking. Furthermore, although operative time had been previously evaluated in adult patients, it remains poorly determined for practice among pediatric cohorts.

Here in the current research, we aimed to identify the risk factors of longer operative time in the pediatric population, which was not directly associated with the surgical morbidity. We further compared postoperative recovery and outcomes between longer or shorter operation duration. To our knowledge, no large study to date has specifically addressed the factors account for the operative time and its correlation to clinical outcomes in pediatric surgical practice.

\section{Materials And Methods}

\section{Patients selection}

We performed retrospective analysis of 810 patients who underwent elective choledochal cysts followed by Roux-en-Y hepaticojejunostomy at the department of general surgery of the affiliated chongqing children's Hospital, Chongqing medical university between August 2010 and Aug 2019. The surgical procedures were performed by five qualified and professional attending surgeons. The inclusion criteria were as follows: patients receiving resection of choledochal cysts and hepaticojejunostomy; open procedure; age $>1$ year and $<10$ years. Exclusion criteria: previous surgical history; coagulopathy or platelet dysfunction; laparoscopic surgery. The institutional review board of the Chongqing Medical University gave expedited approval of this protocol.

\section{Data acquisition}

Data extraction from the electronic medical records included the following aspects: (1) Preoperative variables, including demographic characteristics, clinical parameters and pre-existing comorbidities; (2) Intraoperative data, including American Society of Anesthesiology (ASA) classification, estimated blood loss (EBL), intraoperative hemoglobin levels, intraoperative blood transfusion, operating start time, duration of operation(time from skin incision to complete skin closure); (3) The postoperative outcomes, included the prompt gastrointestinal recovery features, postoperative laboratory profiles, and postoperative complications. Gastrointestinal features were recorded for the first 5 postoperative days, including nausea or vomiting, time to normal diet, abdominal bloating and/or cramps, the first postoperative flatus or defecation, gastric retention. The postoperative complications were ranked according to the Clavien-Dindo classification system. Only grade II complications or higher were recorded in this research, such as postoperative hemorrhage, wound infection, intra-abdominal abscesses,etc. 
According to the median operating time (cutoff value, $165 \mathrm{~min}$ ), patients were dichotomized into short time group (<165 min) and long time group (>165 min) (Table 1) for the purpose of analysis.

Propensity model

The propensity score matching was performed to minimize the biases in baseline characteristics by a multivariable logistic regression model using SPSS 20.0 (IBM, Armonk, NY) or R 3.1.2 (The R Foundation for Statistical Computing). A caliper distance of 0.2 was specified for a 1:1 Propensity scores matching using nearest-neighbor analysis. The linear assumption in PS model was checked using generalized additive model, thus matching $108 \mathrm{HS}$ patients to 108 controls. The characteristics of long time group and a short time group were compared after propensity score matching.

Statistical analysis

The categorical variables, presented as frequencies with percentages, were compared using the chisquared test or Fisher's exact test as appropriate. For normally distributed continuous data, it were expressed as the means \pm (standard deviations) and tested with the Student's t-test or Mann-Whitney U test. For non-normally distributed data, they were expressed as medians (interquartile ranges) and analyzed with the Wilcoxon rank-sum test. The multivariate analysis was performed to determine predictors of operation duration entered the variables with a significance level of $P<0.30$ in univariate analysis. The statistical analyses were performed using the SPSS 20.0 (IBM, Armonk, NY, USA) software package.

\section{Results}

A total of 789 patients who underwent elective Roux-en-Y hepaticojejunostomy between 2009 and 2019 met the inclusion criteria. There were 701 patiens entered into the final analysis for eighty-eight patients exclusion due to unobtainable notes(Table 1).

The factors associated with operating time

In the current study, the average operative time among the entire 701 patients was $165 \mathrm{~min}$, with a coefficient of variation (COV) of $46.9 \%$. The Table 1 summarizes demographic, clinical, and operation features based on the short time and long time operative duration. As indicated, there were no significant differences regarding some preoperative parameters such as gender distribution, ASA classification, prevalence of the hypertransaminasemia and hyperbilirubinemia $(P>0.05)$ (Table 1). Furthermore, some of the preoperative laboratory tests (WBC, $C R P)$ was similar to that of the short-time group $(P>0.05)$. However, patient's deeper fat (greater Weight and BMI) were associated with the duration of operation ( $P$ $<0.01$ ). Interestingly, operations performed in the morning took a longer duration than that in the afternoon. A lager choledochal cyst size, and worse symptoms (Charcot's triad), often resulted in a longer operating time $(P<0.05)$. Moreover, when analyzing individual surgeons, we discovered that the duration 
of operation was associated with surgeon individual performance $(\mathrm{P}<0.01)$ with longer duration for attending surgeon number 5 .

Multivariate Analysis

Three risk factors for the development of longer operating time were identified (Table 4) under multivariable logistic regression analysis. As shown in Table 4, the lager choledochal cyst size, the more likely longer operating time spended $(\mathrm{OR}=1.56 ; 95 \% \mathrm{Cl}, 1.09-2.23 ; \mathrm{p}<0.001)$. Similar results were discovered in the percentage of greater $\mathrm{BMI}(\mathrm{OR}=1.02 ; 95 \% \mathrm{Cl}, 1.00-1.15 ; \mathrm{p}=0.018)$. The patient's age $(\mathrm{OR}=1.17 ; 95 \% \mathrm{Cl}, 1.02-1.39 ; \mathrm{p}=0.012)$ was also significantly associated with longer operating time (Table 2).

\section{Postoperative outcomes}

We performed PS-matching to eliminate systematic differences in baseline characteristics between the patients with short-time group and long-time group. Under PS-matching, 268 patients with short-time group were matched to 268 patients with long-time group. After the PS-matching, the values of standardized mean differences for theincluded continuous and categorical variables reduced and comparable between the two groups (Table 3).

Overall, there were no differences in the number of hypokalemic episodes, intraoperative hypotensive events, metabolic acidosis (defined by low bicarbonate) or other laboratory and hemodynamic parameters between the short-time group and long-time group (Table 4). A trend for accelerated gastrointestinal function recovery was noted in patients with short-time group compared with long-time group, as indicated by the first defecation $(p=0.027)$ and first bowel movement $(p=0.019)$. Furthermore, the ALB values on POD 2-5 were lower in patients who underwent a longer procedure $(P=0.0035)$. Patients with a longer operating time suffered almost equally from vomiting and diarrhea events as patients with a shorter operating time in 5 PODs (Table 4). The incidences of abdominal distention within 5 PODs was marginly higher in the patients with longer operating time compared with the shorter operating time group $(P=0.068)$.

Finally, there were more postoperative complications in patients of long-time group than those with the short-time group, including infectious complications, anastomotic leakage, incision dehiscence, and sepsis, etc. Sixty-two patients $(62 / 268,23.1 \%)$ in the short-time group experienced at least 1 complication compared with $83(83 / 268,31.0 \%)$ in the long-time group, with an odds ratio (OD) of 0.67 (95\% confidence interval [95\% Cl, 0.46-0.99]; $\mathrm{P}=0.026$ ) (Table 4). The mean length of postoperative stay was $7.51 \pm 2.03$ days in patients with a long-time group, which was significantly longer than that $(6.72 \pm 1.54$ days $)$ in patients with a short-time group $(p=0.006)$.

\section{Discussion}


We conducted the present analysis for a pediatric surgical cohort following the same surgical procedure to explore optimized operative duration undergoing abdominal surgery. Current data from three tertiary care hospital suggested that several factors were associated with the operation time, like the patient age, commodity, the mean CBD, etc. As with surgeon's peculiarity, experience and special property contributed to progressively increased operative duration variability. Furthermore, shorter operative duration was associated with significant improvements in recovery measures and postoperative complication. Only disease commodities contribute to both longer operative time and surgical outcome.

The factors led to a long duration of abdominal operations were often complicated and involved in case type, surgeon's proficiency and characterics, emergency operation, and so on [10,11]. The operation duration may be prolonged by unexpected intraoperative findings and operative complexity. The understanding of the risk that delay progression might be in favor to reduce operative duration. The current surgical outcomes, including complication rates, are comparable with those calculated in the previous report $[12,13]$. The pediatric cohort following same surgical procedures originate from the university medical centers was therefore deemed satisfactory to assess the factors influencing operative duration. In this study, we measured the amount of variability in operative duration and identified several factors related to the longer operative duration. After multivariable analysis, the results showed that a lager choledochal cyst size, severe comorbidities, like Charcot's triad independently resulted in a longer operating time. Another factor was patients age, which has not been shown in previous studies to be associated with longer operative time after Roux-en-Y hepaticojejunostomy. the reasons for this association are unclear, but may pertain to alterations in deeper operative surface related to operative visual field exposure that are beyond the scope of this data set. Furthermore, the older patients implies the thicker anastomose, which need the longer operation time to construction. Higher adhesions were also associated with longer operative time, likely reflecting increased complexity of longer cases. We found an relationship between severe commodity and operative time, which likely relates to the chronic inflammation and the resulting impact on the pericyst tissue.

Several studies have shown that the experience and distinctive character of the individual surgeon or the surgical team influences the duration of the operative duration[14, 15, 16]. The current studies illustrate that Operative duration is longer in the presence of junior surgical trainees, which may reflect both the procedural learning curve as well as potential confounding due to referral of complex surgical cases to senior attending supervision. The senior surgeons with increased experience are likely to decrease the duration of procedures. Increased attending supervision could decrease operative duration, however, research is needed in this area. The improvements in operating process efficiency, including an operation team and an anesthesia team can reduce operative duration $[17,18]$. Interestingly, we demonstrated the similar finding that operations in the morning group usually took more time than the afternoon group, which could be the main reason for this finding. The potential impact of surgeons' fatigue and human circadian variations reduced workload requirements on operative experience however, an effect has not been demonstrated so far. 
Longer operation time was a surrogate marker of surgical difficulty, which might be associated with perioperative morbidity and delayed discharge. Although a goal-directed fluid therapy was adopted to control fluid administration, prolonged operative duration may account for excessive intravenous fluid administration and blood loss[19. 20]. In this study, an increased total volume of intraoperative crystalloid and blood loss were presented following operation duration increases. the blood transfusion administration, is an independent predictor of increased morbidity for surgical patients, including gastroenterology patients[21]. Furthermore, fluid accumulation might decline the tissue oxygenation, which was also unfavorable for postoperative gastroenterological recovery and wound healing, the most important reason of the postoperative length of stay[22]. Although an uneventful recovery was ending in most choledochal cyst resection patients. This study identified the remarkable beneficial effects of shorter operative duration for postoperative gastrointestinal recovery of defecation and oral solid diet. In our institute, the clinically intestinal complaints should be adequately monitored as much as possible. In our study, we indeed detected an increased total number of complications and unfavorable postoperative outcomes following an increase in operative duration, which was consistent with previous studies[23]. The longer operative time may relate to prolonged general anesthesia and potential for prolonged catheterization, which should explain some unfavorable postoperative outcomes, like increased urinary tract infection(UTI) risk with urinary retention and respiratory infections. The long operation duration might accelerate the body metabolism and increase the consumption of nutrition, as suggested by previous research[24]. Here in the current research, the postoperative ALB values, which have been widely adopted to evaluate the preoperative immunologic and nutritional status of patients undergoing gastrointestinal and cardiac surgery, significantly increased in the longer operation time group[25, 26]. In the present study for patients with an longer operative time, the average length of stay was longer than the average length of stay in shorter operative time cases in our institution at between 4 and 5 days. The present data highlighted the benefits of low operative duration, which might be associated with postoperative complications in pediatric patients undergoing major surgery. However, while our study supports several factors were associated with prolonged operation time, this was not necessarily associated with postoperative complications. evidence-based best practices should be implemented to promote a safer, higher quality of patient care.

There were several potential limitations, so when interpreting the current data, care should be taken. The retrospective study design with relatively small size of homogeneous patients may limit the generalizability of our conclusions. Moreover, the selection bias could not be fully eliminated although the PS meatching was performed. We were also unable to identify complications or returns to the $O R$ occurring greater than 30 days after the index operations. In addition, it is unlikely that a strict separation the individual contribution of surgeons, that would have prevented us to determine the difference between individual surgeons. It is important for future studies to investigate the effect of each surgeon individually. Finally, it might be interesting to evaluate the effect of surgical nurses, because they are part of the surgical team as well and may affect the operative duration.

\section{Conclusions}


In the current study, we have characterized risk factors, such as patient comorbidities, surgeon's peculiarity, and cyst size, that may predispose to longer operative time. Long operative duration impacts negatively on the postoperative outcomes for pediatric patients with major abdominal operation. Further research is needed to delineate clinical scenarios to optimize preoperative planning and maximize surgical efficiency.

\section{Abbreviations}

ALB: albumin; ASA: American Society of Anesthesiology; BMI: Body Mass Index; CBD: common bile duct; Cl: confidence interval; COV: coefficient of variation; CVP: central venous pressure; EBL: estimated blood loss; OR: odds ratio; PODs: postoperative day; RBC: red blood cells; UTl:urinary tract infection

\section{Declarations}

Ethics approval and consent to participate

This study was approved by the ethics committee of Chongqing Medical University, and was performed in accordance with the Helsinki Declaration of 1975, as revised in 1983. All the patients enrolled were comprehensively informed, and all study participants provided written informed consent prior to enrollment.

Consent to publish

Not applicable.

Availability of data and materials

The datasets during and/or analyzed during the current study are available from the corresponding author on reasonable request.

Competing interests

The authors declare that they have no competing interests.

Funding

This study was supported by the grants from the National Natural Science Foundation of China (No: $30973440,30770950)$ in the design of the study and collection, analysis, and interpretation of data, the Key Project of the Chongqing Natural Science Foundation (CSTC, 2008BA0021, cstc2012jjA0155) for collection, analysis, and interpretation of data and Chongqing Health Planning Commission of Research Fund (No: 2016MSXM044) in writing the manuscript. The funding agency paid for the scholarship of students involved in the research.

Author Contributions 
AL, KG designed and analyzed the data and evaluated the manuscript. QL performed the statistical measurements and analyzed the data. CG, AL analyzed the data and wrote the paper.

Acknowledgements

We thank Prof. Xianqing Jin for providing technical assistance and for insightful discussions during the preparation of the manuscript. We thank Dr Xiaoyong Zhang at the Wistar Institute, USA, for help with the linguistic revision of the manuscript.

\section{References}

1Wang X, Yao Y, Qian H, Li H, Zhu X. Longer Operating Time During Gastrectomy Has Adverse Effects on Short-Term Surgical Outcomes. J Surg Res. 2019 Nov;243:151-159.

2.Zheng HL, Lu J, Zheng CH, Li P, Xie JW, Wang JB, Lin JX, Chen QY, Lin M, Tu RH, Huang CM. Short- and Long-Term Outcomes in Malnourished Patients After Laparoscopic or Open Radical Gastrectomy. World J Surg. 2018 Jan;42(1):195-203.

3.Procter LD, Davenport DL, Bernard AC, Zwischenberger JB. General surgical operative duration is associated with increased risk-adjusted infectious complication rates and length of hospital stay. J Am Coll Surg. 2010 Jan;210(1):60-5.e1-2.

4.Lee MS, Venkatesh KK, Growdon WB, Ecker JL, York-Best CM. Predictors of 30-day readmission following hysterectomy for benign and malignant indications at a tertiary care academic medical center. Am J Obstet Gynecol. 2016 May;214(5):607.e1-607.e12.

5.Gawande AA, Zinner MJ, Studdert DM, Brennan TA. Analysis of errors reported by surgeons at three teaching hospitals. Surgery. 2003 Jun;133(6):614-21.

6.Dexter F, Epstein RH, Schwenk ES. Tardiness of starts of surgical cases is not substantively greater when the preceding surgeon in an operating room is of a different versus the same specialty. $\mathrm{J}$ Clin Anesth. 2019 Mar;53:20-26.

7.van Eijk RP, van Veen-Berkx E, Kazemier G, Eijkemans MJ. Effect of Individual Surgeons and Anesthesiologists on Operating Room Time. Anesth Analg. 2016 Aug;123(2):445-51.

8.Dull MB, Gier CP, Carroll JT, Hutchison DD, Hobbs DJ, Gawel JC. Resident impact on operative duration for elective general surgical procedures. Am J Surg. 2017 Mar;213(3):456-459.

9.Evans C, Lim J, Gatzen C, Huang A. Factors influencing laparoscopic colorectal operative duration and its effect on clinical outcome. Surg Laparosc Endosc Percutan Tech. 2012 Oct;22(5):437-42.

10.Cheng H, Chen BP, Soleas IM, Ferko NC, Cameron CG, Hinoul P. Prolonged Operative Duration Increases Risk of Surgical Site Infections: A Systematic Review. Surg Infect (Larchmt). 2017 
Aug/Sep;18(6):722-735.

11.Cheng H, Clymer JW, Po-Han Chen B, Sadeghirad B, Ferko NC, Cameron CG, Hinoul P. Prolonged operative duration is associated with complications: a systematic review and meta-analysis. J Surg Res. 2018 Sep;229:134-144.

12.Xia HT, Liu Y, Yang T, Liang B, Wang J, Dong JH. Better long-term outcomes with hilar ductoplasty and a side-to-side Roux-en-Y hepaticojejunostomy. J Surg Res. 2017 Jul;215:21-27.

13.Xia HT, Dong JH, Yang T, Liang B, Zeng JP. Selection of the surgical approach for reoperation of adult choledochal cysts. J Gastrointest Surg. 2015 Feb;19(2):290-7.

14. Weigl M, Antoniadis S, Chiapponi C, Bruns C, Sevdalis N. The impact of intra-operative interruptions on surgeons' perceived workload: an observational study in elective general and orthopedic surgery. Surg Endosc. 2015 Jan;29(1):145-53.

15. Heslin MJ, Doster BE, Daily SL, Waldrum MR, Boudreaux AM, Smith AB, Peters G, Ragan DB, Buchalter $\mathrm{S}$, Bland KI, Rue LW. Durable improvements in efficiency, safety, and satisfaction in the operating room. J Am Coll Surg. 2008 May;206(5):1083-9;

16.Azzi AJ, Shah K, Seely A, Villeneuve JP, Sundaresan SR, Shamji FM, Maziak DE, Gilbert S. Surgical team turnover and operative time: An evaluation of operating room efficiency during pulmonary resection. J Thorac Cardiovasc Surg. 2016 May;151(5):1391-5.

17.Kelz RR, Tran TT, Hosokawa P, Henderson W, Paulson EC, Spitz F, Hamilton BH, Hall BL. Time-of-day effects on surgical outcomes in the private sector: a retrospective cohort study. J Am Coll Surg. 2009 Oct;209(4):434-445.e2.

18.Kelz RR, Freeman KM, Hosokawa PW, Asch DA, Spitz FR, Moskowitz M, Henderson WG, Mitchell ME, Itani KM. Time of day is associated with postoperative morbidity: an analysis of the national surgical quality improvement program data. Ann Surg. 2008 Mar;247(3):544-52.

19.Tan X, Liu J, Guo C. Crystalloid Administration Is Associated with the Recovery of Pediatric Elective Roux-en-Y Hepaticojejunostomy. Eur J Pediatr Surg. 2019 Jan 21. doi: 10.1055/s-0038-1677544.

20.Zhu H, Chen B, Guo C. Aggressive crystalloid adversely affects outcomes in a pediatric trauma population. Eur J Trauma Emerg Surg. 2019 Apr 27. doi: 10.1007/s00068-019-01134-0.

21.Johnson DJ, Scott AV, Barodka VM, Park S, Wasey JO, Ness PM, Gniadek T, Frank SM. Morbidity and Mortality after High-dose Transfusion. Anesthesiology. 2016 Feb;124(2):387-95.

22.Hao F, Guo H, Zhong J, Geng Q, Yang Y, Chen B, Guo C. Effects of Prostaglandin E1 on Patients Undergoing Major Gastrointestinal Surgery. Ann Surg. 2018 Apr;267(4):759-765. 
23. Short HL, Fevrier HB, Meisel JA, Santore MT, Heiss KF, Wulkan ML, Raval MV. Defining the association between operative time and outcomes in children's surgery. J Pediatr Surg. 2017 Oct;52(10):1561-1566.

24.Wakita M, Fukatsu A, Amagai T. Nutrition assessment as a predictor of clinical outcomes for infants with cardiac surgery: using the prognostic nutritional index. Nutr Clin Pract. 2011;26:192e198.

25.Nozoe $\mathrm{T}$, Kohno $\mathrm{M}$, Iguchi $\mathrm{T}$, et al. The prognostic nutritional index can be a prognostic indicator in colorectal carcinoma. Surg Today. 2012;42:532e535.

26. Wierdak M, Pisarska M, Kuśnierz-Cabala B, Witowski J, Dworak J, Major P, Małczak P, Ceranowicz P, Budzyński A, Pędziwiatr M. Changes in plasma albumin levels in early detection of infectious complications after laparoscopic colorectal cancer surgery with ERAS protocol. Surg Endosc. 2018 Jul;32(7):3225-3233.

\section{Tables}

Table 1. The factors associated with operating time 


\begin{tabular}{|c|c|c|c|}
\hline & \multicolumn{3}{|l|}{ Total Population } \\
\hline & $<165$ minutes $(348)$ & $>165$ minutes(353) & $\mathrm{p}$ Values \\
\hline Age (yrs) & $2.29 \pm 1.32$ & $2.95 \pm 1.87$ & 0.058 \\
\hline Female: Male & 93(26.7) & $89(25.2)$ & 0.41 \\
\hline Weight (kg) & $11.88 \pm 4.86$ & $12.97 \pm 5.28$ & 0.035 \\
\hline BMI, median (range) & $24(20-29)$ & $26(22-32)$ & 0.023 \\
\hline \multicolumn{4}{|l|}{ Laboratory findings } \\
\hline hypertransaminasemia, n (\%) & $225(64.7)$ & $236(66.9)$ & 0.30 \\
\hline hyperbilirubinemia, n (\%) & 138(39.7) & $143(40.5)$ & 0.44 \\
\hline $\mathrm{WBC}(109 / \mathrm{L})$, mean $\pm \mathrm{SD}$ & $9.15 \pm 3.54$ & $9.06 \pm 3.96$ & 0.17 \\
\hline CRP (mg/L, normal value: 0-8), mean \pm SD & $12.68 \pm 4.31$ & $12.78 \pm 4.67$ & 0.29 \\
\hline \multicolumn{4}{|l|}{ Ultrasound presentation } \\
\hline Mean CBD, cm, mean \pm SD & $1.95 \pm 0.76$ & $2.64 \pm 1.69$ & 0.0012 \\
\hline Charcot's triad, n (\%) & $56(16.1)$ & $89(25.2)$ & 0.02 \\
\hline \multicolumn{4}{|l|}{ Operating start time } \\
\hline Morning & $95(27.3)$ & $126(35.7)$ & 0.010 \\
\hline Afternoon & $253(72.7)$ & $227(64.3)$ & \\
\hline \multicolumn{4}{|l|}{ ASA classification } \\
\hline ASA1-2 & $237(68.1)$ & $252(71.4)$ & 0.19 \\
\hline ASA3-4 & $111(31.9)$ & $101(28.6)$ & \\
\hline \multicolumn{4}{|l|}{ Individual surgeons, n (\%) } \\
\hline Attending1 & $72(20.7)$ & $76(21.5)$ & 0.43 \\
\hline Attending2 & $65(18.7)$ & $61(17.3)$ & 0.35 \\
\hline Attending3 & 108(31.0) & $79(22.4)$ & 0.006 \\
\hline Attending 4 & $46(13.2)$ & $49(13.9)$ & 0.44 \\
\hline Attending5 & $57(16.4)$ & $88(24.9)$ & 0.003 \\
\hline
\end{tabular}

Table 2. Multivariate analysis of related factors for longer operating time.

\begin{tabular}{l|l|l|l|}
\hline & OR & $95 \%$ CI & $\mathrm{p}$ \\
\hline Age (yrs) & 1.17 & $(1.02-1.39)$ & 0.012 \\
\hline choledochal cyst size & 1.56 & $(1.09-2.23)$ & $<0.001$ \\
\hline BMI & 1.02 & $(1.00-1.15)$ & 0.018 \\
\hline
\end{tabular}


Table 3. The inclusion variables in the PS-matching analysis

\begin{tabular}{|c|c|c|c|}
\hline & \multicolumn{3}{|l|}{ Total Population } \\
\hline & $<165$ minutes $(268)$ & >165 minutes $(268)$ & $\mathrm{p}$ Values \\
\hline Age (yrs) & $2.42 \pm 1.26$ & $2.51 \pm 1.39$ & 0.26 \\
\hline Female: Male & $87(37.5)$ & 85(39.9) & 0.41 \\
\hline Weight (kg) & $12.13 \pm 4.42$ & $12.24 \pm 4.75$ & 0.19 \\
\hline BMI, median (range) & $25(22-27)$ & $25(22-28)$ & 0.32 \\
\hline \multicolumn{4}{|l|}{ Laboratory findings } \\
\hline hypertransaminasemia, n (\%) & $175(65.3)$ & $176(65.7)$ & 0.38 \\
\hline hyperbilirubinemia, n (\%) & 108(40.3) & $111(41.4)$ & 0.46 \\
\hline WBC $(109 / L)$, mean \pm SD & $9.11 \pm 3.36$ & $9.09 \pm 3.54$ & 0.32 \\
\hline CRP (mg/L, normal value: $0-8)$, mean \pm SD & $12.68 \pm 3.67$ & $12.72 \pm 3.88$ & 0.37 \\
\hline \multicolumn{4}{|l|}{ Ultrasound presentation } \\
\hline Mean CBD, $\mathrm{cm}$, mean $\pm \mathrm{SD}$ & $2.12 \pm 0.68$ & $2.26 \pm 1.14$ & 0.24 \\
\hline Charcot's triad, n (\%) & $43(16.0)$ & $44(16.4)$ & 0.49 \\
\hline \multicolumn{4}{|l|}{ Operating start time, n (\%) } \\
\hline Morning & $81(30.2)$ & $85(31.7)$ & 0.45 \\
\hline Afternoon & 187(69.8) & 183(68.3) & \\
\hline \multicolumn{4}{|l|}{ ASA classification, n (\%) } \\
\hline ASA1-2 & $186(69.4)$ & 183(68.3) & 0.37 \\
\hline ASA3-4 & $82(30.6)$ & $85(31.7)$ & \\
\hline
\end{tabular}

Table 4. Outcome characteristics in the matched population with operative times above and below 165 minutes 


\begin{tabular}{|c|c|c|c|c|}
\hline & $<165$ minutes(268) & $>165$ minutes $(268)$ & p Values & Odds ratio $(95 \% \mathrm{CI})$ \\
\hline Hypotensive events, n(\%) & $26(9.7)$ & $31(11.6)$ & 0.29 & \\
\hline Norepinephrine usage, $\mathrm{n}(\%)$ & $38(14.2)$ & $41(15.3)$ & 0.40 & \\
\hline Furosemidum, n(\%) & $24(9.0)$ & $27(10.1)$ & 0.38 & \\
\hline Metabolic acidosis, n(\%) & $8(3.0)$ & $11(4.1)$ & 0.32 & \\
\hline Nadir of hemoglobin(g/L) & $27(10.1)$ & $38(14.2)$ & 0.093 & \\
\hline \multicolumn{5}{|l|}{ Operative blood loss (mL) } \\
\hline Intraoperative transfusion, $\mathrm{n}(\%)$ & $31(11.6)$ & $44(16.4)$ & 0.067 & \\
\hline Albumin on POD 2-5, mean $\pm \mathrm{SD}$, (g/L, normal range, 35-50) & $31.25 \pm 5.95$ & $28.64 \pm 6.83$ & 0.008 & $1.12(1.01-1.56)$ \\
\hline First bowel movement, days, Mean \pm SD & $2.28 \pm 0.91$ & $2.81 \pm 0.76$ & 0.019 & $0.56(0.38-0.98)$ \\
\hline Abdominal distension, $\mathrm{N}(\%)$ & $26(9.7)$ & $38(14.2)$ & 0.068 & $0.65(0.38-1.11)$ \\
\hline Diarrhea, N (\%) & $13(4.9)$ & $21(7.8)$ & 0.11 & \\
\hline Vomiting, N (\%) & $24(9.0)$ & $32(11.9)$ & 0.16 & \\
\hline Removal nasogastric tube (days) & $3.17 \pm 0.39$ & $3.35 \pm 0.47$ & 0.51 & \\
\hline First defecation (days) & $2.54 \pm 1.36$ & $3.36 \pm 1.48$ & 0.027 & $0.69(0.31-1.07)$ \\
\hline No. of patients with complications, $\mathrm{n}(\%)$ & $62(23.1)$ & $83(31.0)$ & 0.026 & $0.67(0.46-0.99)$ \\
\hline Total number of complications, n(\%) & $73(27.2)$ & $94(35.1)$ & 0.031 & $0.69(0.48-1.00)$ \\
\hline postoperative stay (d) & $6.72 \pm 1.54$ & $7.51 \pm 2.03$ & 0.006 & $0.72(0.42-0.96)$ \\
\hline
\end{tabular}

